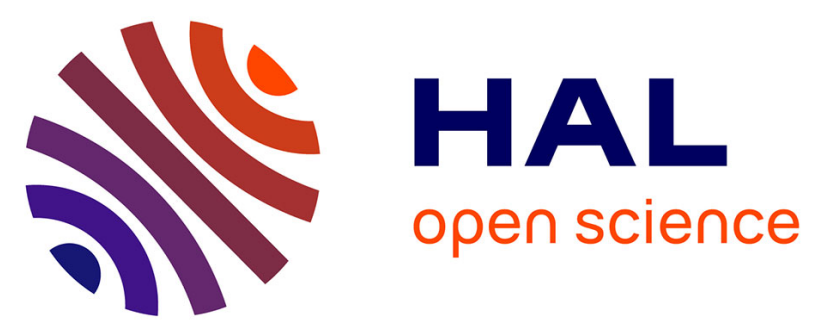

\title{
13C NMR investigation of the linear and nonlinear dynamics of the spin-density wave of (TMTSF)2PF6
}

E. Barthel, G. Kriza, G. Quirion, P. Wzietek, D. Jérome, J. Christensen, M. Jørgensen, K. Bechgaard

\section{- To cite this version:}

E. Barthel, G. Kriza, G. Quirion, P. Wzietek, D. Jérome, et al.. 13C NMR investigation of the linear and nonlinear dynamics of the spin-density wave of (TMTSF)2PF6. Journal de Physique IV Proceedings, 1993, 03 (C2), pp.C2-231-C2-234. 10.1051/jp4:1993246 . jpa-00251329

HAL Id: jpa-00251329

https://hal.science/jpa-00251329

Submitted on 1 Jan 1993

HAL is a multi-disciplinary open access archive for the deposit and dissemination of scientific research documents, whether they are published or not. The documents may come from teaching and research institutions in France or abroad, or from public or private research centers.
L'archive ouverte pluridisciplinaire HAL, est destinée au dépôt et à la diffusion de documents scientifiques de niveau recherche, publiés ou non, émanant des établissements d'enseignement et de recherche français ou étrangers, des laboratoires publics ou privés. 


\title{
${ }^{13} \mathrm{C}$ NMR investigation of the linear and nonlinear dynamics of the spin-density wave of (TMTSF) ${ }_{2} \mathbf{P F}_{6}$
}

\author{
E. BARTHEL, G. KRIZA, G.QUIRION, P. WZIETEK, D. JÉROME, J.B. CHRISTENSEN*, \\ M. JØRGENSEN* ${ }^{*}$ and K. BECHGAARD*
}

Laboratoire de Physique des Solides, Associé au CNRS, Université de Paris-Sud, 91405 Orsay cedex, France

${ }^{*}$ CISMI, University of Copenhagen, Blegdamsvej 21, 2100 Copenhagen, Denmark

\begin{abstract}
The interpretation of our recent measurements of the temperature dependence of the NMR spinlattice relaxation rate in the spin-density wave (SDW) state in (TMTSF) ${ }_{2} \mathrm{PF}_{6}$, as well as of our combined conduction noise and NMR spectra measurements in the sliding SDW state is discussed in relation with the prominent features of the electric response. Our measurements suggest that the phason effective mass is equal to the band mass, and show that the condensate density is not strongly reduced, as has been proposed. Consequences on the dynamics of the phason, and especially the so-called pinned mode resonance, are discussed.
\end{abstract}

In spin-density waves (SDW) as in charge-density waves (CDW), the presence of an incommensurate density modulation driven by a Fermi surface instability induces special dynamic properties. In an incommensurate system, indeed, there appears a specific low-frequency collective excitation mode, called phason, which is the Goldstone mode associated with the broken translation symetry. Clear manifestations of phason dynamics, which are well known in CDW's, have recently been evidenced in the electric response of SDW's in the Bechgaard salts :

- non linear dc conductivity [1], with generation of conduction noise in the sliding state [2],

- low-frequency microwave resonance mode [3],

- large low-frequency dielectric constant [4].

One of the main goal of the recent investigations of the SDW dynamics is therefore to point out and explain differences with CDW's.

In SDW's, the electronic modulation does not induce a lattice distorsion, so that the effective mass $m^{*}$ of the phason is not expected to be enhanced, unlike CDW's. In this respect, the small oscillator strength found in the microwave resonance mode [3] is difficult to understand. In contrast to superconductors, momentum conservation is crucial to the existence of the SDW, because the broken symetry is the translation symetry. Therefore, as in a clean superconductor [5], no absorption should be observed above the gap frequency for a SDW with $m^{\star}=m_{b}$, where $m_{b}$ is the band mass, and the total oscillator strength should be in the collective mode. It was therefore suggested that the phason effective mass is large in the SDW, or that the number of electrons implied is reduced [4]. 


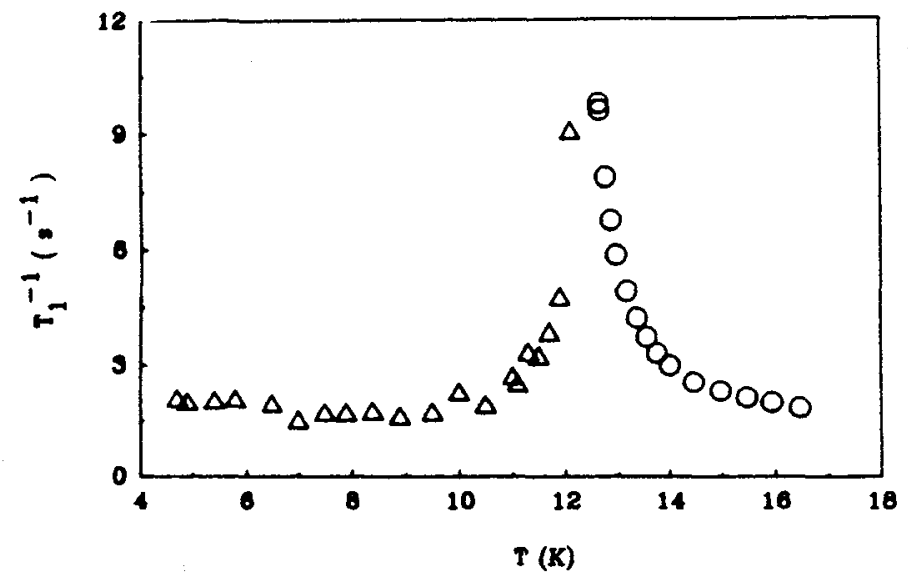

Temperature dependence of the NMR spin-lattice relaxation rate in (TMTSF) ${ }_{2} \mathrm{PF}_{6}$ (from Ref.[15]). The peak is due to the critical fluctuations at the transition. The temperature independence of the relaxation rate below the transition is typical of phason fluctuations.

Another question which has aroused some interest is the origin of the conduction noise. Two mechanisms of generation have been proposed :

- the noise is generated in the bulk of the sample, through the temporal modulation of the pinning potential [6],

- the noise is generated at the contacts when normal electrons are converted to condensed pairs [7].

In CDW's, these two mechanisms are expected to produce the same fundamental frequency for the conduction noise, because the pinning potential periodicity is also the electronic periodicity. However, in SDW"s. the wavelength of the pinning potential is twice shorter than the modulation wavelength [8]. A simple picture to explain this result is that the SDW consists in two CDW's out of phase by $180^{\circ}$. Since the pinning mechanism depends only on the charge and is nonlinear, the period of the pinning potential is half of the SDW period. As a result, we expect that the frequency of the noise generated by the bulk mechanism is twice larger than the frequency generated by the contact mechanism.

In this contribution. we will suggest how NMR investigations can deal with these questions. NMR has long proved to be a powerful tool in the study of incommensurate systems. It was used in distorsive crystals [9]. In CDW's, in the nonlinear conductivity regime, it has proved and characterised the motion of the modulation [10] as will be further discussed below. In the SDW of (TMTSF) ${ }_{2} \mathrm{PF}_{6}$, it has shown the antiferromagnetic nature of the ground state, proved the incommensurability of the SDW [11], and demonstrated the motion of the condensate in the nonlinear regime [12]. In addition, a sharp decrease of the NMR relaxation rate below $3.5 \mathrm{~K}$ has evidenced a controversial transition [13].

We first discuss the phason contribution to the low-frequency linear response. The very large value of the dielectric constant $\left(\simeq 10^{9}\right)$ is due to a specific manifestation of the phason. This mode may be described, in a simple picture, as a deformation of the modulation between two pinning centers, thus producing a charge dipole [14]. Damping of the phasons results in the decrease of the dielectric constant above a given cut-off frequency [15]. This frequency is strongly temperature dependent in the range $2-4 \mathrm{~K}$.

Another measurement of the linear response is provided by the NMR relaxation rate. As is well known, spin $1 / 2$ nuclei like ${ }^{13} \mathrm{C}$ relax through magnetic fluctuations only. Moriya [16] has proved that the relaxation rate $T_{1}^{-1}$ is then proportional to $k_{b} T / \hbar \omega \sum_{q} \chi^{\prime \prime}(q, \omega)$, where $T$ is the temperature, $\omega$ the nuclear Larmor frequency and $\chi$ the magnetic susceptibility. In a previous paper [17], we have shown that the experimental high magnetic field spin-lattice relaxation is independent of temperature between 4 and $10 \mathrm{~K}$ (figure). Two kinds of excitations are expected to contribute to the relaxation : phasons and magnons. However, the large 
magnetic field introduces a gap, estimated at $12 \mathrm{~K}$ for our $9.3 \mathrm{~T}$ field, in the magnon dispersion relation, so that the only significant contribution comes from the phasons. To our knowledge, this is the only evidence for phason dynamics in the magnetic response, since antiferromagnetic resonance probes magnon excitations. Using the Moriva formula and previous results by $\mathrm{H}$. Schulz [18], Girard et al. [19] have shown theoretically that in the model of an undamped phason, $T_{1}^{-1}$ is proportional to $T^{1-\mu}$, where $\mu=m_{b} / m^{\star}$. In this model, we conclude from our data that $m^{\star}=m_{b}$, as expected for SDW's. Although no dielectric constant data is available above $4 \mathrm{~K}$, we conclude from the extrapolated temperature and frequency dependences $[4,15]$ that the dielectric constant must be large at $10^{8} \mathrm{~Hz}$ (our Larmor frequency) in the temperature range 4-10 K. NMR relaxation rate and dielectric constant therefore probe the same mode, and their comparison should yield a better insight in the validity of this theoretical approach:

- this calculation is valid if the thermal correlation length is smaller than an ill-defined pinning length. In a deformation mode picture, the large value of the dielectric constant certainly supports this hypothesis.

- the effect of the damping is more difficult to assess because it is not included in the calculation and because its behaviour above $4 \mathrm{~K}$ is unknown. It may lead to a different temperature dependence.

We hope that this question will soon be solved, and a reliable value of the phason effective mass determined.

The nature of the SDW state below $3.5 \mathrm{~K}$ has led to a controversy. Because the magnetoresistance oscillations vanish at about this temperature $[20]$, and the NMR relaxation rate under pressure, just below the pressure-induced superconducting transition, exhibits a peak [21], it was suggested that there is a phase transition with a change of wave vector, leading to the destruction of electron pockets. On the other hand, Trætteberg et al. observed a change in the temperature dependence of the nonlinear conductivity in the alloys $(\mathrm{TMTSF})_{2}\left(\mathrm{SbF}_{6}\right)_{x}\left(\mathrm{AsF}_{6}\right)_{(1-x)}[22]$. The cross over temperature extrapolates to about $4 \mathrm{~K}$ for the pure sample. These authors conclude that a cross over in the damping mechanism lies at the root of the temperature dependence of both non-linear transport and NMR relaxation rate. Unfortunately. no dielectric constant data are available in this temperature range. It is therefore quite clear that an NMR relaxation rate investigation of the alloyed samples should produce interesting conclusions. We note that the disappearance of electron pockets may lead to a change in the damping mechanism, so that both positions may be compatible.

We now turn to the sliding of the SDW. The power of combined NMR spectra, conduction noise, and nonlinear conductivity measurements in characterizing a sliding modulation has been demonstrated in CDW's by Berthier and coworkers [10]. The nonlinearity of the dc conductivity is a measure of the current carried by the motion of the SDW. As already discussed, the conduction noise frequency is proportional to the sliding velocity, but in SDW's, the proportionality constant depends on the a priori unknown generation process. Moreover, the narrowing of the NMR line is a direct measurement of the sliding velocity: when the SDW is static, the NMR spectrum is broadened by the spatial distribution of local fields; when the SDW slides, there results an averaging of the local fields, and narrowing of the NMR line. Such a set of data therefore enables one to measure the charge carried by the sliding motion (hence the condensate density), and the proportionality constant between conduction noise and sliding velocity.

Our experimental results in the sliding SDW of. (TMTSF) ${ }_{2} \mathrm{PF}_{6}$ have been presented elsewhere [23]. From the analysis of the data, we conclude that the condensate density is not substantially smaller than expected. Similar results have been obtained by Clark et al. [24]. From the direct comparison of the noise and NMR spectra obtained for the same values of current on the same sample, we have established that in the SDW of (TMTSF) ${ }_{2} \mathrm{PF}_{6}$ as in CDW's, the conduction noise generation process is of the contact type. We note that the opposite result has been obtained by Clark et al., from the analysis of the echo amplitude oscillations at low current and the slope of the mean noise frequency at a somewhat higher current.

In conclusion, our NMR experiments suggest that the phason effective mass is actually equal to the band mass. We have also proved that the condensate density is as large as theoretically expected. It is therefore very unlikely that the resonance peak in the absorption at $5 \mathrm{GHz}$ is due to a simple pinned mode resonance. From our characterization of the nonlinear transport, we conclude that the noise generation process in SDW's as in CDW's is of the contact type. Finally, we are quite confident that the NMR investigation of 
the alloyed samples will contribute to the understanding of the behaviour of the SDW of (TMTSF) ${ }_{2} \mathrm{PF}_{6}$ below $3.5 \mathrm{~K}$.

We thank J.C. Ameline and G. Tévanian for their help during the experiment, and C. Berthier for usefu] comments. E. B. was supported by the Etudes Doctorales de l'Ecole Polytechnique.

\section{References}

[1] S. Tomić et al., Phys. Rev. Lett. 62, 462 (1989).

[2] K. Nomura et al., Solid State Commun. 72, 1123 (1989); N. Hino et al., Synth. Metals 40, 275 (1991);

G. Kriza et al., Phys. Rev. Lett. 66, 1922 (1991).

[3] D. Quinlivan et al., Phys. Rev. Lett. 65, 1816 (1990).

[4] G. Mihály, Yong Kim et G. Grüner, Phys. Rev. Lett. 66, 2806 (1991).

[5] D.C. Mattis and J. Bardeen, Phys.Rev 111, 412 (1958).

[6] G. Gruner et al., Phys.Rev.Lett. 46, 511 (1981).

[7] N. P. Ong, G. Verma, and K. Maki, Phys. Rev. Lett. 52, 663 (1984).

[8] I.I. Tutto et A. Zawadowski, Phys. Rev. Lett. 60, 1442 (1988).

[9] R. Blinc, Phys.Rep. 79, 331 (1989).

[10] P. Butaud et al., J.Physique (France) 51, 59 (1990).

[11] J.M. Delrieu et al. , J.Phys.(France) 47, 839 (1986); T.Takahashi et al. , J.Phys.Soc.Jpn 55, 1364 (1986).

[12] J.M. Delrieu and K. Kinoshita, Synth. Metals 43, 3947 (1991); W. H. Wong et al., Phys. Rev. Lett. 70, 1882 (1993).

[13] T. Takahashi et al., Synth. Met. 19, 225 (1987).

[14] P.B. Littlewood, Phys. Rev. B 36, 3108 (1987).

[15] O. Trætteberg et al., Synth. Met. 55, to be published.

[16] T. Moriya, Progress of Theoretical Physics 16, 23 (1956).

[17] E. Barthel et al., Europhys. Lett. 21, 87 (1993).

[18] H. Schulz, J.Phys. C 16, 6769 (1983).

[19] M. Girard, E. Barthel and C. Bourbonnais, to be published.

[20] J.P. Ulmet et al., J. Phys. Lett. (Paris) 46, L535 (1985).

[21] T. Takahashi, Synth. Met. 27, B397 (1988).

[22] O. Trætteberg et al., submitted.

[23] E. Barthel et al., submitted.

[24] W.G. Clark et al., to be published. 